\title{
Paper
}

\section{Variable Gain PID Control of Ultrasonic Motor using Novel Hybrid PSO with Improved Searching Ability}

\author{
Shota NakAShima* ${ }^{\dagger}$ Member, \\ SHENGLIN MU $\quad$ Non-member, \\ KANYA TANAKA ${ }^{\text {II } \quad \text { Non-member }}$ \\ SHINGO ARAMAKI ${ }^{\dagger}$ Non-member \\ HUIMIN LU $\mathrm{U}^{\S} \quad$ Non-member
}

(Received September 7, 2018, revised December 9, 2018)

\begin{abstract}
The ultrasonic motor (USM) is a type of actuator driven by frictional force. At present, because of their excellent features, USMs are used in a range of applications, such as for autofocus of cameras and in actuators for devices in MRI environments and micro robots. However, because the dynamic characteristics of USMs vary according to temperature, humidity, and load conditions, their mathematical modeling is difficult. Thus, PID control has conventionally been used for the control of USMs. However, conventional PID control with fixed gains cannot compensate for the non-linear characteristic variation of a USM. In this research, we propose a novel hybrid particle swarm optimization (PSO) system for USM control. The proposed method uses PSO to automatically tune the optimized PID gains. The experiments demonstrated that optimized gains in USM control can be obtained in real time, and high accuracy.
\end{abstract}

Keywords: Ultrasonic motor, PID control, Particle swarm optimization, Random inertia weight PSO, Real-time control

\section{Introduction}

The ultrasonic motor (USM) is a new type of motor, which is driven by frictional force. It differs from conventional electronic motors in that the main components are a rotor and a stator. When a high frequency alternating voltage is applied to the piezoelectric element on the stator of a USM, mechanical vibration is generated in the stator. The resulting frictional force between the rotor and the stator, which is produced by the ultrasonic wave generated from the vibration, drives the rotor in a certain direction. The USM has many advantages, including electromagnetic compatibility and high torque. USMs are also silent while running, compact, lightweight, have a quick response, and can run at low speeds with no gearing requirements. USMs are therefore used in various applications such as for autofocus of cameras and in actuators of devices in MRI environments and micro robots [1] [4]. Since USMs are driven by frictional force, their dynamic characteristics vary according to different conditions. As a result, this makes mathematical modeling based on physical analysis becomes difficult [5]. PID control has therefore been used for the control of USMs in previous research [6] [8]. However, PID control

\footnotetext{
* Corresponding: s-naka@yamaguchi-u.ac.jp

$\dagger$ Graduate School of Sciences and Technology for Innovation, Yamaguchi University

Yamaguchi University, 2-16-1, Tokiwadai, Ube-city, Yamaguchi, 7558611, Japan

¥ Graduate School of Science and Engineering, Ehime University

$\S$ Department of Mechanical and Control Engineering, Kyushu Institute of Technology

II Department of Electronics and Bioinformatics, Meiji University
}

with fixed gains cannot compensate for the changes in the dynamics of a USM, which is caused by temperature or humidity changes or by a load change. This means that the control of USM deteriorates under such changes, and optimization of the three PID gains $K_{P}, K_{I}$, and $K_{D}$ is necessary [9] [14].

In this research, we used PSO to tune the PID gains in order to achieve high performance of USM control [15]. PSO does not require USM modelling or the use of differential information for optimization. Moreover, since PSO is effective in non-linear optimization, we applied our proposed method to non-linear changes of characteristics. The effectiveness of the proposed method was experimentally verified.

This paper is organized as follows. In "Particle swarm optimization" section, we describe the PSO algorithm. "Novel hybrid PSO" section discusses the proposed novel hybrid PSO with improved searching ability. "Machine experiments" sections present the experimental results. Finally, in "Conclusions" section, we give our conclusions.

\section{Particle swarm optimization}

PSO was introduced by Eberhart and Kennedy in 1995 through simulations of a simplified social model [16]. It is a new population-based probability-like optimization algorithm inspired by social behavior. Its algorithm mimics the social actions of a swarm (e.g., of birds, fish, or insects) to find the optimized solution in a search space. The basic concept of PSO can be explained as follows. In a swarm, each particle searches for an optimal solution in the search space. 


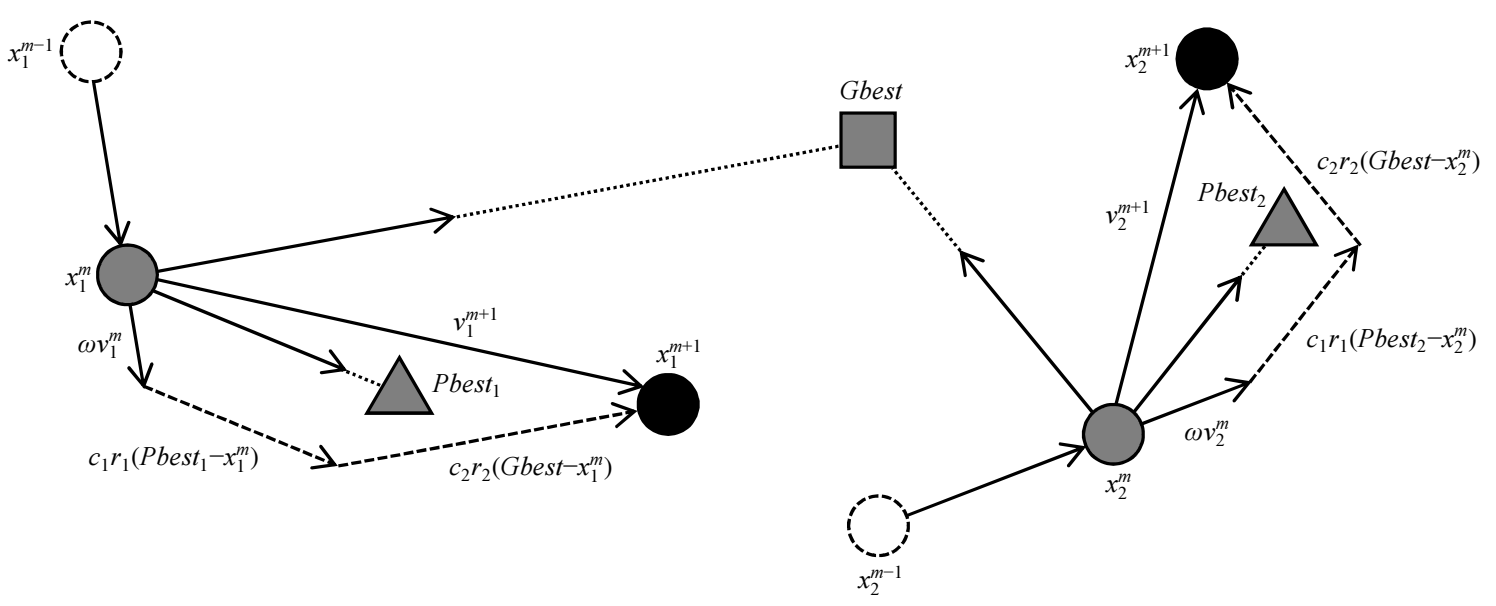

Figure 1: Movement of particle in PSO.

Also, each particle carries information about its velocity and position. This information is shared between particles in the swarm. Based on this shared information, the position and velocity of the particles can be updated as they converge on the optimal solution. Since solutions are evaluated by a fitness function, there is no requirement for continuity or for differential information when using a PSO. In addition, PSO is considered simpler than genetic algorithms, and is effective in non-linear optimization. The fundamental principle of PSO is explained as follows. Each particle in multidimensional space has a position vector and a velocity vector. The particle memorizes the best position it has achieved, as determined by the evaluation function, and stores its fitness value. The particle that has the local best position imparts positional information to the particle swarm in its neighborhood. Then, the information of the best position among all the particles is imparted to the whole swarm.

Here, we consider optimization to maximize the fitness function $f: M \rightarrow M^{\prime} \subset R$ with respect to the variable $x \in M \subset R^{n}$. The particle $N$ exists in spatial position $M$. At a number of repetitions $m(=0,1,2, \cdots)$ for the $j(=0,1,2, \cdots, N)$-th particle, the position vector and the velocity vector become $x_{j}^{m}$ and $v_{j}^{m}$. Pbest is the point at which the evaluation function $f(x)$ is maximized at the search point $x_{j}^{1}, x_{j}^{2}, \cdots, x_{j}^{m}$ of each particle in the PSO. Gbest is the point at which $f(x)$ is maximized at the search point of the whole swarm. Pbest shows the best position that any individual particle has discovered so far, and the best position that the whole swarm has discovered so far. From this information, the PSO updates position the vector $x_{j}^{m}$ and velocity vector $v_{j}^{m}$ of the individual particles.

The updating of the position and velocity of each particle is repeated using the following recurrence formulae:

$$
\begin{aligned}
v_{j}^{m+1}=\omega v_{j}^{m}+c_{1} r_{1}\left(\text { Pbest }_{j}-x_{j}^{m}\right) & \\
& +c_{2} r_{2}\left(\text { Gbest }-x_{j}^{m}\right)
\end{aligned}
$$

where $\omega$ represents the inertia weight, and $r_{1}$ and $r_{2}$ are random numbers drawn from a uniform distribution of interval $[0,1]$; these are generated in each component. $c_{1}$ and $c_{2}$ are positive constants, called cognitive and social scaling parameters, respectively (usually, $c_{1}=c_{2}$ ). $c_{1}$ and $c_{2}$ show the weight coefficient with respect to the search for the best position of each particle and of the whole particle swarm. The particle's movement using Equation 1 and Equation 2 is illustrated in Figure 1. Velocity and position are updated in a PSO for a two-dimensional space. The movement of particles is governed by three factors: (1) the inertial part, $\omega v_{j}^{m}$; (2) the cognitive part, $\left(\right.$ Pbest $\left._{j}-x_{j}^{m}\right)$; and (3) the social part, $\left(\right.$ Gbest $\left.-x_{j}^{m}\right)$. The velocity vector of $v_{j}^{m+1}$ is based on the three vectors, as shown in Equation 1.

In this research, we consider the solution space $M_{P I D} \subset$ $R^{3}$ is $K_{P}$ axis, $K_{I}$ axis, and $K_{D}$ axis of the PID gains in the PID control. This solution space $M_{P I D}$ is a threedimensional coordinate space. Each particle of the PSO expresses it as a point in solution space $M_{P I D}$. We used this to apply optimization by PSO.

\section{Novel hybrid PSO}

The inertia weight $\omega$ is an important parameter in balancing the local area search ability and the wide area search ability of a particle. Generally, if the value of the inertia weight is large, the particle performs a wide area search of the solution space. In contrast, if the value of the inertia weight is small, the particle performs a local area search of the solution space. Accordingly, the technique for changing the inertia weight and coordinating the search ability of a PSO is called the inertia weight approach (IWA) [17] [18]. Linearly decreased inertia weight PSO, which is a linear decrease function, and nonlinearly decreased inertia weight PSO (NDW-PSO) [19], which is a non-linear decrease function, are both given in $\omega$ and are used for a conventional IWA. These algorithms decrease $\omega$ based on a function that decreases to minimum $\omega_{\min }$, which is set optionally from a maximum $\omega_{\max }$, which we also set optionally. Thus, the particle performs a wide area search at the early stages of search and a local area search at the final stages of search. NDW-PSO can change the ratio between the wide area search and the local area search using the function. However, there is a problem with this algo- 
rithm, in that the local area search decreases at the early stages of search, and the wide area search decreases at the final stages of search. Consequently, the particle may fall into a local solution. This tendency is particularly high in a dynamic environment. Moreover, as the strategy of weight updating of all particles is uniform, additional searching, which may delay convergence, has to be implemented even for the particles around the optimal.

Therefore, in this research, we used a novel hybrid PSO (NH-PSO) [20] to improve searching ability. NH-PSO combines the advantages of an NDW-PSO and of a random inertia weight PSO (RIW-PSO). RIW-PSO in particular is superior in its search ability. The proposed algorithm compares the evaluation value Fitness ${ }_{j-1}^{m}$ of the previous particle with the evaluation value Fitness ${ }_{j}^{m}$ of the current particle in the NDW-PSO. If the evaluation value Fitness ${ }_{j}^{m}$ of the current particle decreases, RIW-PSO is applied to particle $x_{j}^{m+1}$ of the same particle number in the next repetition $m+1$. Therefore, this algorithm can compensate for load changes in the final stages of search, and the local optimization problem is avoided.

Fitness $_{j}^{m}$ was derived using Equation 3.

$$
\text { Fitness }_{j}^{m}=\frac{1}{1+\sum_{k=0}^{T}\{e(k)\}^{2}}
$$

where $T$ is the expected calculation-time. In our experiment, we used $T=10 \mathrm{~ms}$. The proposed algorithm compared the evaluation value Fitness $s_{j-1}^{m}$ of the previous particle with the evaluation value Fitness ${ }_{j}^{m}$ of the current particle using Equation 3. When Fitness $s_{j-1}^{m} \leq$ Fitness $_{j}^{m}$, Equation 4 was used. When Fitness ${ }_{j-1}^{m}>$ Fitness $_{j}^{m}$, Equation 5 was employed. In this way, the parameter $\omega$ was set in the proposed algorithm.

$$
\begin{aligned}
& \omega=\left(\omega_{\max }-\omega_{\min }\right)\left(\frac{m_{\max }-m}{m_{\max }}\right)^{\tau}+\omega_{\min } \\
& \omega=\left(\omega_{\max }-\omega_{\min }\right) r+\omega_{\min }
\end{aligned}
$$

where $r$ in Equation 5 represents random numbers drawn from a uniform distribution of interval $[0,1]$; $\tau$ in Equation 4 is a parameter called a nonlinear index number. The value of the nonlinear index number will determine the degree of nonlinear path of the decreasing inertia weight $\omega$. The local search ability will be increased if the value of the nonlinear index is large, and the global search ability will be decreased. The influence of the nonlinear index number is shown in Figure 2.

When applying the proposed method, in which the most suitable gains change according to the characteristic variation, the searching method will switch to RIW-PSO, improving searching ability. In this way, the NH-PSO follows the most suitable solution, allowing efficient optimization of a particle.

A PID controller using NH-PSO is shown in Figure 3. $K_{P}, K_{I}$, and $K_{D}$ of each PID gain are optimized using a NHPSO algorithm.

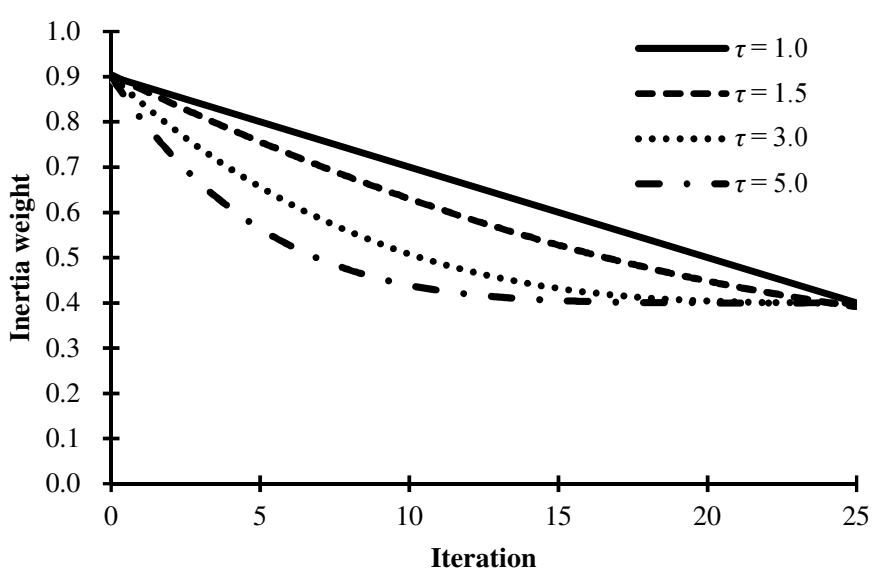

Figure 2: The impact of nonlinear index number.

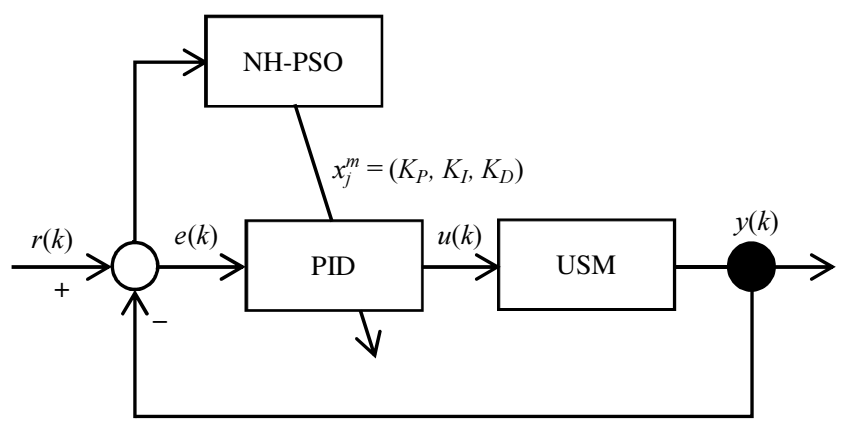

Figure 3: NH-PSO PID controller.

\section{Machine experiments}

4.1 Experimental conditions We performed an actual machine experiment to confirm the usefulness of $\mathrm{NH}$ PSO. The USM servo system used in the experiment worked as follows. The USM, the electromagnetic brake, and the encoder were connected on the same axis. The angular positional information from an encoder was sent to a Personal Computer (PC) through a counter board. In the $\mathrm{PC}$, an error signal was calculated based on the difference between the target value and the angular positional information from the encoder. A control signal was then calculated based on the error signal and was sent to the driver circuit through an I/O board. Finally, the USM was operated as intended.

The target setting was the following square wave with a period of $4 \mathrm{sec}$, an amplitude of $45 \mathrm{deg}$, and a 10 cycle movement period. The initial gains of the PID controller were $K_{P}=1.3, K_{I}=70$, and $K_{D}=0.0001$. The PSO parameters were set as follows: (1) the particle number, $N=5$; (2) the cognitive constant, $c_{1}=1.0$; (3) the social constant, $c_{2}=1.0$; (4) the maximum value of the inertia weight, $\omega_{\max }=0.9$; $(5)$ the minimum value of inertia weight, $\omega_{\min }=0.4$; and (6) the nonlinear index number, $\tau=1.5$. The specifications of the USM servo system are given in Table 1.

4.2 Experimental results The steady-state error was measured in 20 times. Figure 4 and Figure 5 show a histogram of the results, and the positional accuracy of the USM in unloaded and loaded conditions. The transverse 


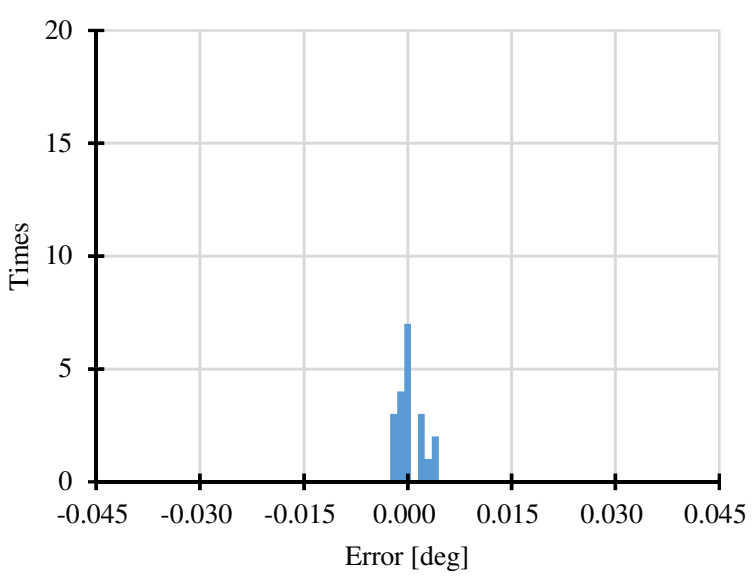

(a) NDW-PSO.

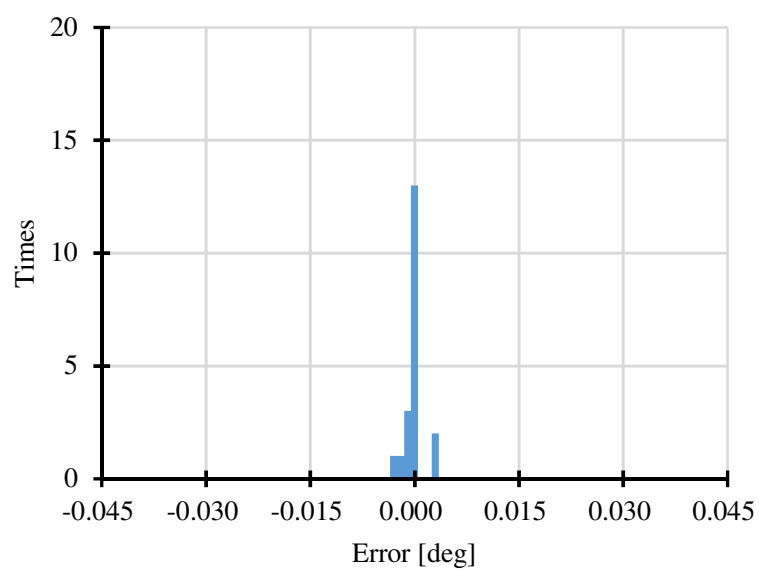

(b) NH-PSO.

Figure 4: Positional accuracy of USM (unloaded).

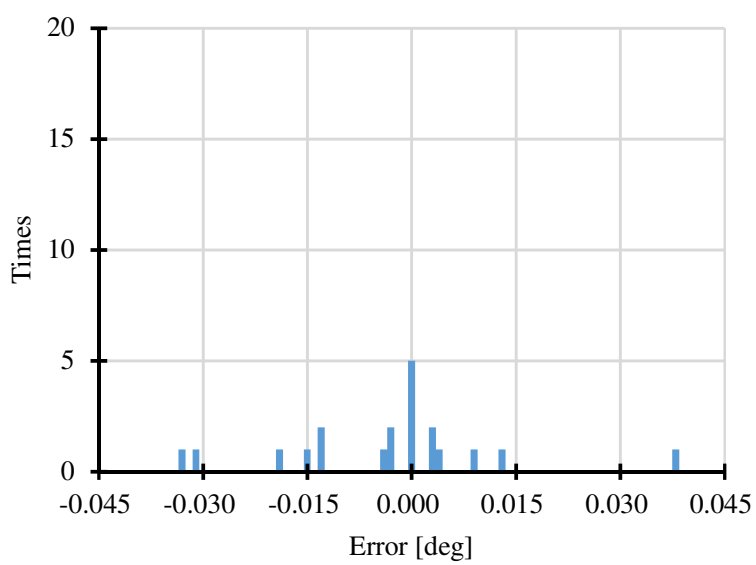

(a) NDW-PSO.

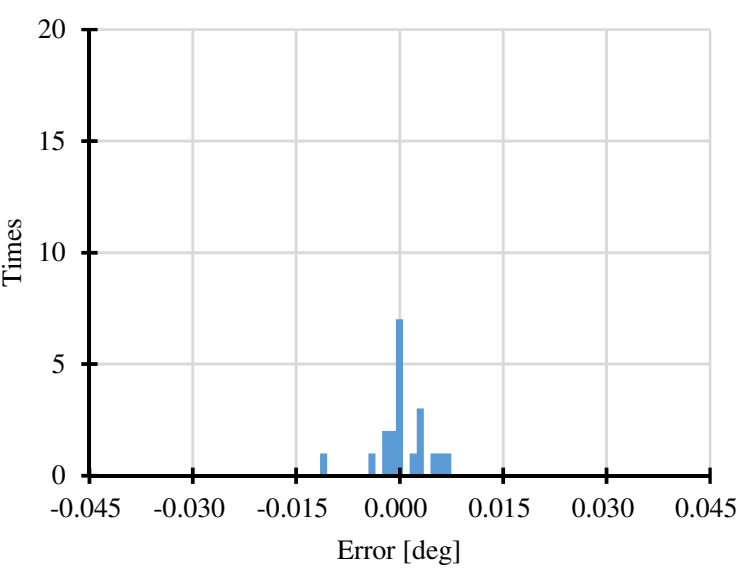

(b) NH-PSO.

Figure 5: Positional accuracy of USM (loaded).

Table 1: Specifications of USM, encoder, and load.

\begin{tabular}{ll}
\hline \multirow{2}{*}{ USM } & Rated rotation speed: $100 \mathrm{rpm}$ \\
& $\begin{array}{l}\text { Rated torque: } 0.5 \mathrm{Nm} \\
\text { Holding torque: } 1.0 \mathrm{Nm}\end{array}$ \\
\hline Encoder & Resolution: $0.0011^{\circ}$ \\
\hline
\end{tabular}

of the graph indicates the size of the error. The value of the steady-state error measured an error before $10 \mathrm{~ms}$ when the sign of the target input signal was switched. We were able to confirm that the error of the NH-PSO in the proposed method was lesser than that of the NDW-PSO of the traditional technique in both the unloaded and the loaded conditions.

Consequently, the NH-PSO of the proposed method compensated for the non-linear change of motion of the USM. We also confirmed that the control performance of the proposed method was better than that of the traditional method.

The variation of PID gains using the PSO is shown in Figure 6 to Figure 8. It is clear that three gains tuned by the proposed NH-PSO converged to the optimal range close to the optimal obtained by the NDW-PSO. The convergence of the proposed method was achieved with fewer iterations (around 500) than the NDW-PSO (more than 1000). The fitness comparison is shown in Figure 9. It can be seen that the fitness of the two methods converged to a value around 1. Compared to the fitness variation of a traditional NDWPSO, the proposed NH-PSO had higher and more durative fitness. Meanwhile, as can be seen in Figure 10, the inertia weight was kept to the optimal random number in the proposed method, which kept the performance in USM control. The experimental results suggest that, compared with the traditional method, the proposed method improved search ability in the optimization of PID tuning. The experiment demonstrated that the proposed method performs well in avoiding local optima and in compensating the characteristics of the USM in control.

\section{Conclusions}

In this paper, to improve the searching ability of an intelligent control method for a USM, a NH-PSO algorithm was proposed. The PID control method for USM in the pro- 


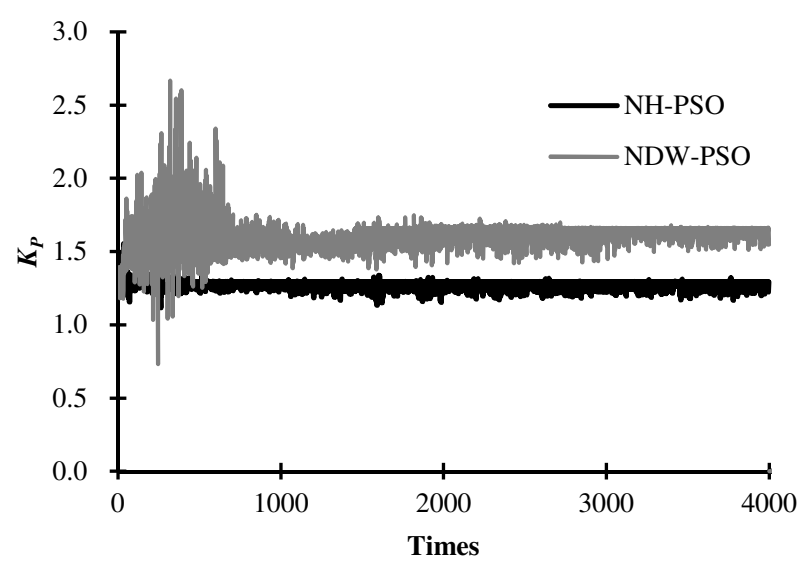

Figure 6: Convergence of $K_{P}$.

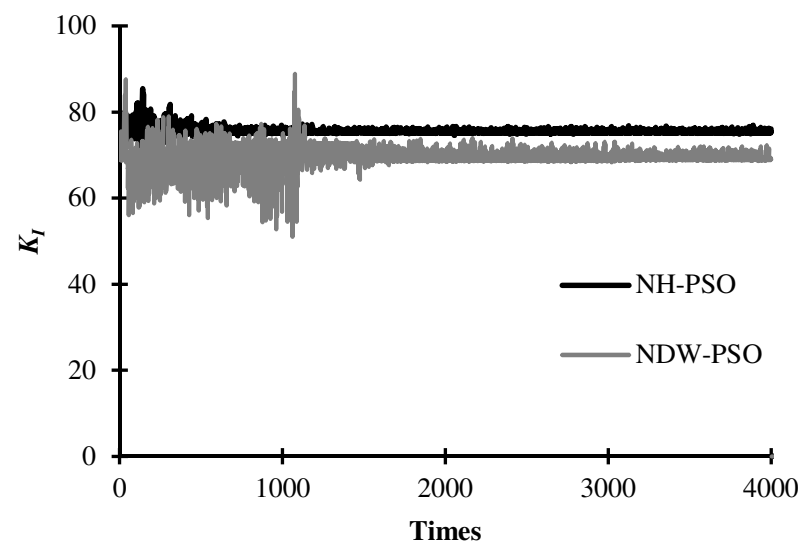

Figure 7: Convergence of $K_{I}$.

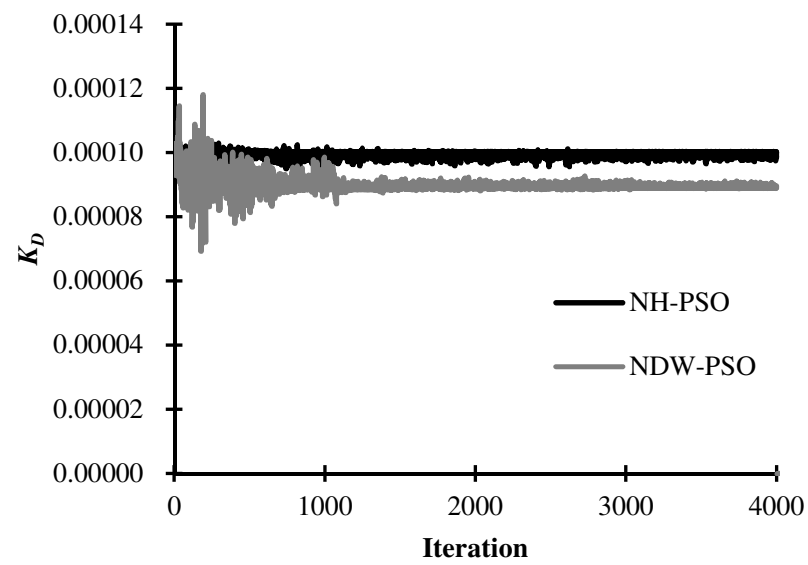

Figure 8: Convergence of $K_{D}$.

posed algorithm has an advanced ability to compensate for the nonlinearity in USM control. To test the control performance of the proposed method, a group of experiments were conducted. Compared with the NDW-PSO method introduced in previous research, the proposed method offered better positioning results in both no-load and load conditions. In the proposed method, the PID gains were tuned automatically online. The effectiveness of the proposed method was confirmed, and it has been demonstrated that USM control can be achieved with high accuracy by applying the proposed method.

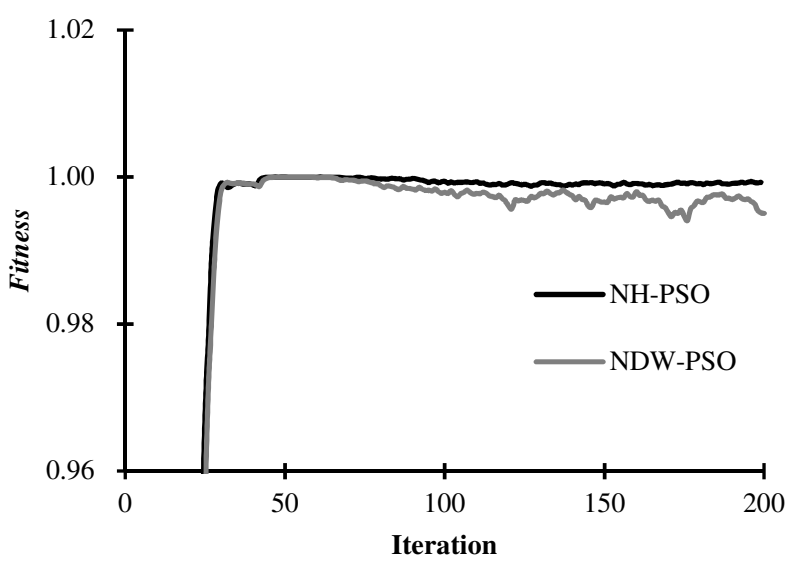

Figure 9: Convergence of Fitness.

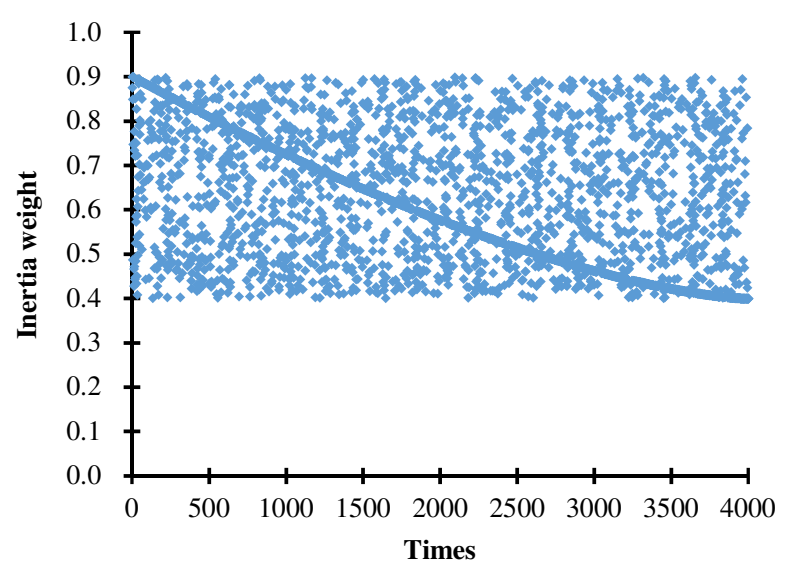

Figure 10: Convergence of inertia weight.

\section{References}

[1] Kenji Uchino, "Piezoelectric ultrasonic motors: overview", Smart Materials and Structures, Vol.7, No.3, pp.273-285, 1998.

[2] Valentinas Snitka, "Ultrasonic actuators for nanometre positioning”, Ultrasonics, Vol.38, No.1-8, pp.20-25, 2000.

[3] Toshiyuki Aodai, Jun Izawa, Takahiro Shimizu, Koji Ito and Shigeki Toyama, "Development of MR Compatible Manipulandum for Finger Movements", Journal of the Japan Society for Precision Engineering, Vol.74, No.4, pp.405-410, 2008.

[4] Ikuo Yamano and Takashi Maeno, "Five-fingered Robot Hand using Ultrasonic Motors and Elastic Elements", Proceedings of the 2005 IEEE International Conference on Robotics and Automation, pp.2673-2678, 2005.

[5] Tomonobu Senjyu, Mitsuru Nakamura, Naomitsu Urasaki, Hideomi Sekine and Toshihisa Funabashi,"Mathematical Model of Ultrasonic Motors for Speed Control", Electric Power Components and Systems, Vol.36, No.6, pp.637-648, 2008.

[6] Koji Fukuda, Takuya Kamano, Takashi Yasuno and Takayuki Suzuki, "Two-degree-of-freedom Servo System with Ultrasonic Motor by Frequency and Phase Fuzzy Hybrid Control", IEEJ Transactions on Electronics Information and Systems, Vol.119-C, No.11, pp.1310-1315, 1999.

IIAE Journal, Vol.7, No.1, 2019 
[7] Kanya Tanaka, Masato Oka, Akihiko Uchibori, Youichirou Iwata and Hiroshi Morioka, "Precise Position Control of Ultrasonic Motor Using PID Controller Combined with NN", IEEJ Transactions on Electronics, Information and Systems, Vol.122-C, No.8, pp.1317-1324, 2002.

[8] Kenji Nishibori, Shigeru Okuma, Yasuo Eryu and Satoru Sakai, "Position Control of Robot Manipulators with Ultrasonic Motors using Pulse Width Modulation and Fuzzy Controllers", Transactions of the Japan Society of Mechanical Engineers Series C, Vol.60, No.574, pp.2052-2056, 1994.

[9] Tomonobu Senjyu, Hiroshi Miyazato, Satoru Yokoda and Katsumi Uezato", "Speed control of ultrasonic motors using neural network", IEEE Transactions on Power Electronics, Vol.13, No.3, pp.381-387, 1998.

[10] Tomonobu Senjyu, Satoru Yokoda and Katsumi Uezato, "peed control of ultrasonic motors using fuzzy neural network", Journal of Intelligent and Fuzzy Systems, Vol.8, No.2, pp.135-146, 2000

[11] Kanya Tanaka, Yuji Wakasa, Takuya Akashi and Masato Oka, "PI control adjusted by GA for ultra-sonic motor", SIEEJ Transactions on Industry Applications, Vol.127, No.6, pp.59-65, 2007.

[12] Kanya Tanaka, Yoshie Yoshimura, Yuji Wakasa and Takuya Akashi, "Variable Gain Type Internal Model Control-PID Speed Control for Ultrasonic Motors", IEEJ Transactions on Industry Applications, Vol.128, No.12, pp.1326-1332, 2008.

[13] Shenglin Mu, Kanya Tanaka, Yuji Wakasa, Takuya Akashi, Nobuaki Kobayashi, Shigeru Uchikado and Yasuhiro Osa, "Intelligent IMC-PID control for ultrasonic motor", 2009 International Conference on Networking, Sensing and Control, pp.201-205, 2009.

[14] Shenglin Mu and Kanya Tanaka, "Position control of ultrasonic motor using PID-IMC combined with neural network based on probability", International Journal of Applied Electromagnetics and Mechanics, Vol.41, No.1, pp.59-71, 2013.

[15] Yuji Wakasa, Kanya Tanaka, Takuya Akashi and Yuki Nishimura, "PSO-based simultaneous tuning method for PID controllers and dead-zone compensators and its application to ultrasonic motors", International Journal of Innovative Computing, Information and Control", Vol.6, No.10, pp.4593-4604, 2010.

[16] James Kennedy and Russell Eberhart, "Particle swarm optimization", IEEE International Conference on Neural Networks - Conference Proceedings, Vol.4, pp.1942-1948, 1995.

[17] Alrijadjis Djoewahir, Kanya Tanaka and Shota Nakashima, "Adaptive PSO-based self-tuning PID controller for ultrasonic motor", International Journal of Innovative Computing, Information and Control, Vol.9, No.10, pp.3903-3914, 2013.

[18] Alrijadjis Djoewahir, Kanya Tanaka and Shota Nakashima, "Hybrid strategy for improving PSO and its application for self-tuning PID controller on position control of ultrasonic motor", 2013 International Conference of Information and Communication Technology (ICoICT), pp.99-104, 2013.
[19] Alrijadjis Djoewahir, Kanya Tanaka and Shenglin Mu, "A modified particle swarm optimization with nonlinear decreasing inertia weight based PID controller for ultrasonic motor", International Journal of Innovation Management and Technology, Vol.3, No.3, pp.198-201, 2012.

[20] Shingo Aramaki, Motoki Tanaka and Shenglin Mu and Shota Nakashima and Kanya Tanaka, "Variable Gain PID Control of Ultrasonic Motor Using a Novel Hybrid PSO with Improved Searching Ability", Proceedings of the 3rd International Conference on Industrial Application Engineering 2015, pp.118-123, 2015

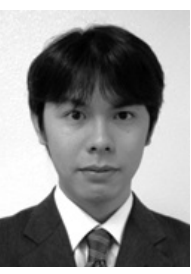

Shota Nakashima (Member) received a Ph.D. degree in Electrical Engineering from Kyushu Institute of Technology in 2010. From 2010 to 2012, he was an Assistant Professor at Ube National College of Technology. Currently, he is a Senior Assistant Professor at Yamaguchi University. His current research interests include image processing, intelligent sensing, and artificial life. $\mathrm{He}$ is a member of IEEE, SCIE, IEEJ and IIAE.

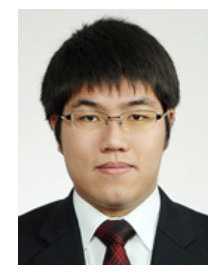

Shingo Aramaki (Member) was born in Fukuoka, Japan on January, 1991. He received his B.S. degree from Nishinippon Institute of Technology, Japan, in 2014 and his M.S. degree from Graduate School of Science and Engineering, Yamaguchi University, Japan, in 2016. Currently, he is a Ph.D. student in Graduate School of Sciences and Technology for Innovationk, Yamaguchi University. His current research interests include image processing, intelligent sensing, and intelligent control.

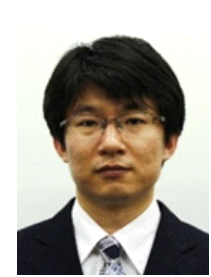

Shenglin Mu (Member) received a B.E. degree in Mechanical Engineering and Automation from the Northeastern University, China, in 2007, and M.E. and Ph.D. degrees in Electronic and Information Systems Engineering from the Yamaguchi University, Japan, in 2010 and 2013, respectively. In 2013, he joined the Department of Electronic Control Engineering, National Institution of Technology, Hiroshima College. He is currently with the Graduate School of Science and Engineering, Ehime University. His current research interests include control engineering, esp. intelligent control; mechatronics; robotics; and sensing technology. $\mathrm{He}$ is a member of IEEE, IEEJ, and SICE.

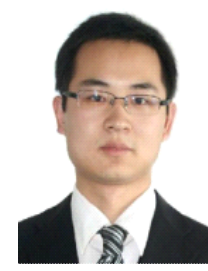

Huimin Lu (Member) received a B.S. degree in Electronics Information Science and Technology from Yangzhou University in 2008. He received M.S. degrees in Electrical Engineering from Kyushu Institute of Technology and Yangzhou University in 2011. He received a Ph.D. degree in Electrical Engineering from Kyushu Institute of Technology in 2014. From 2013 to 2016, he was a JSPS research fellow at Kyushu Institute of Technology. Currently, he is an Assistant Professor in Kyushu Institute of Technology and an Excellent Young Researcher of MEXT-Japan. His research interests include computer vision, robotics, artificial intelligence, and ocean observing. 


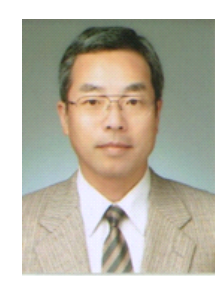

Kanya Tanaka (Member) graduated from the science and engineering program at the $\mathrm{Na}-$ tional Defense Academy in 1982. From 1990 through 1992, he was associate professor of electronic engineering at Hiroshima Denki Institute of Technology. From 1992 through 1998, he was an associate professor of mechanical engineering at Ehime University. He is currently a professor in the Graduate School of Sciences and Technology for Innovation at Yamaguchi University. His main interests are in the theory of intelligent control and related applications. 